\title{
DATA MINING FOR CUSTOMER QUERIES IN ERP MODEL - A CASE STUDY
}

\author{
Abdullah S. Al-Mudimigh, Zahid Ullah, Farrukh Saleem, Fahad N. Al-Aboud \\ King Saud University, Riyadh \\ Kingdom of Saudi Arabia \\ mudimigh@ksu.edu.sa, zahid@ksu.edu.sa, farrukh800@ksu.edu.sa, albod99@yahoo.com
}

\begin{abstract}
Customers always play a key role for the establishment or mean of crisis for any organization. In this paper, we applied data mining techniques on MADAR data for the perfection and development of the organization as well as making their customers more and more satisfied and contented. In the presented model we specially kept customers on the top and emphasized and highlighted the role of customers for every organization. By using their characteristics and surroundings we clustered the data on the basis of action taken against the raised question. In addition, the clustered data employed on the Apriori algorithm and finally, we discovered new rules and patterns from the database for formulating the process in adequate and satisfactory milieu. For the best implementation we used two data mining techniques; Clustering and Association Mining, to get most valuable, informative and strong results for this organization. This is the way to have the best association and gratify their customer in future.
\end{abstract}

Key Words: CRM, Data Mining, Clustering, Apriori.

\section{INTRODUCTION}

CRM has different meanings, It is a business strategy to select and manage customer to optimize long-term value, CRM also is a strategy that increases the importance of relationship marketing and integrates with other organization strategies $[1,3]$.

Despite the efforts of CRM on efficiency and affectivity of management decisions, statistics reflect that unreasonable decisions are made by CRM systems; IT experts said errors were caused by incorrect or not enough data [2].

The core of the ERP system circulates within the company as well as the management information and control needs of the entire production process, including reducing inventory, labor, and operation costs, improving business processes to enhance operation efficiency and improving customer response [4]. However, Markus and Robey (1988) pointed out that although industry specific ERP has already focused on industry characteristics and includes the optimal business operation management model, the promotion of ERP is still significantly related to interaction with the organization [4]. Beyond that CRM often describes a strategic or philosophic approach for managing customers [9]. Hence CRM could be seen from a process oriented, technological, capability-oriented, philosophical, and/or strategic perspective [9]. According to Forrester Research, 57\% of business firms cannot justify CRM investments because they cannot measure customer profitability [9]. The necessary broad level of security is determined by the CRM security objectives which an organization needs to meet[10]. A CRM security strategy outlines in general terms how an organization will achieve its CRM security objectives [10].

In order to increase the use of ERP systems it is recommended to begin with the financial section, the applications invoicing, cost control, accounting and financial then it should be added many functions from Financial, Relation Management Production, Distribution, e- Business and Analyses [5]. The order of the customer is routing automatically to the next department when one department finishes their work of the customer order and each department have access to the single database that holds the customer's new order [6].

\section{EXPERIMENTAL MODEL}

The presented model shown in the Fig.1, described the abstract of all concerned departments exist in every organization. We generally divided the structure of an organization in three layers. Each layer has its own departments and officials having 
specific responsibilities and burden. This is basically the combination of CRM and ERP which are essential part of the enterprise. We enhanced this abstract by adding knowledge discovery layer, where the rule generator plays the special and critical role to generate new rules and patterns from the large database by using data mining techniques (Clustering, Association, Classification). We implemented our model on MADAR (Customer Based Organization) data, by using clustering and Apriori algorithms for the generation of new rules and recommended these rules for the future perspective of MADAR. According to the MADAR environment whenever a customer request engender directly forward to the proper department of CRM Layer for the assessment and positive response. CRM works in front of any organization after receiving the query it will redirect to the ERP layer for the appraisal and configuration of these queries. After statistical analysis and evaluation of the request the answer back to the corresponding customer and this feedback will be saved in the database for the future requirements. Now the work start in knowledge discovery view which select some of the useful data from the large database for its work.

\section{DESCRIPTION OF THREE LAYERS}

\subsection{CRM Layer}

Whenever a customer contact with the company the customer support officer receive customer's request. In the company's prospectus this department has much importance because of correspondence directly with the customer. In our model we presented customer relationship management as an outer view. CRM is responsible for receiving requests and replying to the customer directly. These requests includes queries, complaints, suggestions and orders then forward these requests to the inner view enterprise resource planning (ERP) through the query generator. After taking action on the perspective request the answer will forward through the CRM Layer. And result will also be saving in the database for knowledge discovery view.

\subsection{ERP Layer}

The important part of the model is ERP Layer. In this view each department have equal access to a single database that holds the customer's data or complaints. In this layer the customer queries rotating and evaluating by the concern department. For example a customer contact with customer support department (CRM layer) after initial review and statistics this query will throw to the ERP Layer. The ERP's department is responsible to find the solution and give proper reply to the customer and forward feedback back to customer through CRM layer as well as in the central database for future assistance. 


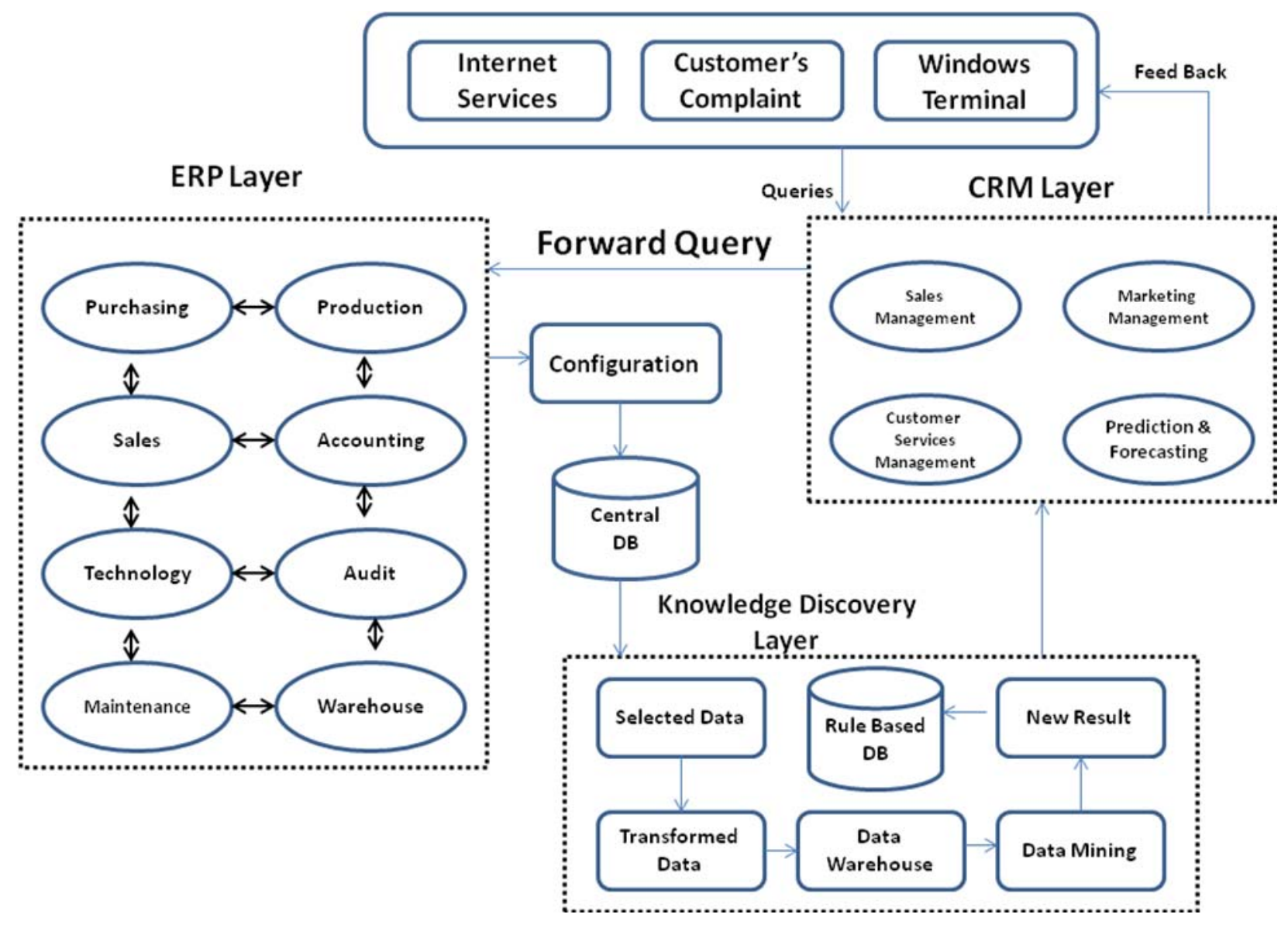

Fig. 1- ERP - CRM Model

\subsection{Knowledge Discovery Layer}

Knowledge discovery can be defined as the extraction of contained, hidden and useful information from the large database. As per the definition, in the presented model this layer is also concern with the central database having all kind of data posted from any department of ERP and CRM layers. The data has several attributes and characteristics according to the queried customer. As the specialty of this layer we used Clustering and Apriori algorithms for advantageous and high-quality results.

\section{FINDINGS AND RESULTS}

For the practical implementation of presented model we applied data mining technique on MADAR data.
MADAR is an ERP based organization working in King Saud University dealing with all administrative software of the university and also do work for outer projects. The efficient implementation of presented ERP-CRM model using data mining techniques is applied on MADAR data. First we applied clustering on the data thereafter For this we used Association mining- Apriori Algorithm for finding new rules and patterns from the experienced data. The description of all phases on our case study is as follows:

\subsection{Programming Tool Used}

For the implementation of Apriori Algorithm to get new rules on the MADAR data we used VB technology. Input and output data files can be in any format e.g databases, Notepad, Excel worksheets, etc. But in this case we used Notepad and Excel worksheet as input and output files. 


\subsection{Data Clustering}

Data clustering is a method in which we make cluster of objects that are somehow similar in characteristics. The criterion for checking the similarity is implementation dependent. Clustering is often confused with classification, but there is some difference between the two. In classification the objects are assigned to pre defined classes, whereas in clustering the classes are also to be defined $[12,14]$. The goal of data clustering, also known as cluster analysis, is to discover the natural grouping(s) of a set of patterns, points, or objects [13].

\subsubsection{Clustering Implementation}

Clustering implementation on the data is a process in which we try to make some groups of objects together having similar characteristics according to the pre-defined criteria. The criteria to merge similar objects in one group are depending on the implementation. We clustered the data on the basis of actions of the customer's query. Moreover, action's column has again four (04) types of action have taken against the particular query. The similarity criterion which we set here in this case is dependent on the action. We described these actions as numbers 1,2,3,4 for gathering them in separated groups. As the result we got four different clusters, in which each cluster is fulfilling the selection criteria shown in Fig.2. Cluster's evaluation graph is shown in Fig.3.

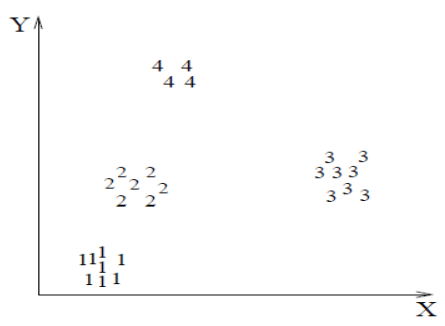

Fig. 2- Data Clusters

\subsection{Apriori Algorithm}

Apriori is a classic algorithm for learning association rules. Apriori is designed to operate on databases containing transactions (for example, collections of items bought by customers, or details of a website frequentation) $[7,8]$. As is common in association rule mining, given a set of itemsets (for instance, sets of retail transactions, each listing individual items purchased), the algorithm attempts to find subsets which are common to at least a minimum number of the itemsets. Apriori uses a "bottom up" approach, where frequent subsets are extended one item at a

\subsubsection{Apriori Implementation}

According to the Fig. 5, four clusters have been generated on the basis of selection criteria. These

\section{Cluster's Evaluation}

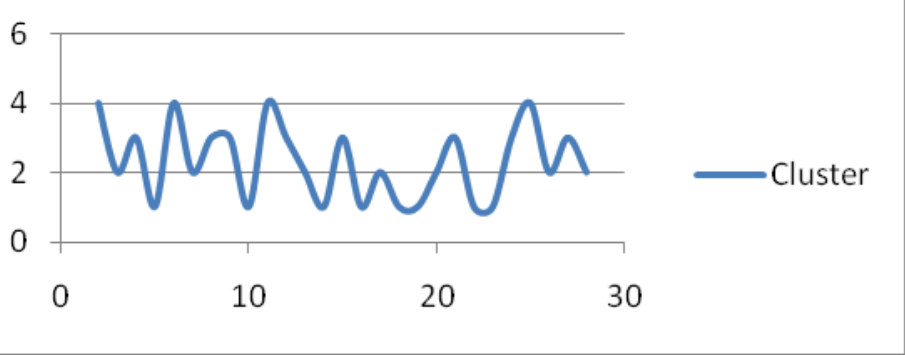

Fig. 3- Cluster's Evaluation Graph time (a step known as candidate generation), and groups of candidates are tested against the data. The algorithm terminates when no further successful extensions are found $[7,8,11]$. One way to construct a simpler model computed from data, easier to understand and with more predictive power is to create a set of simplified rules [11]. Apriori Algorithm is suitable to compute the rules and patterns and predict for any organization to improve the customer satisfaction. We implement Apriori algorithm on MADAR data and generated some rules and patterns for MADAR.

clusters we took as an input for the Apriori implementation to generate new rules. This is called data mining on mined data because we used two best data mining algorithm for acquiring the best solution and rules. First clustering and then on clustered data 
we employed Apriori algorithm on it. For the Apriori implementation we compared all the clustered with each other to find out the subset of all clusters shown in Fig. 4.

\section{Selected Data for Input File - Notepad}

File Edit Format View Help

Bugs in software-A, Bugs in software-B, Bugs in software-c, Bugs in softwai

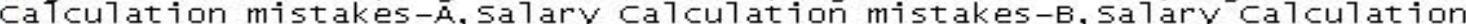

Changes in contracts for new employee-B, changes in contracts for

new emp $70 y e e-c$, sa $7 a r y$ structure for each designation-s, sajary

structure for each designation-B, salary structure for each

designation-c, Budget approval for coming year-A, Budget approval

for comjing year-B, Budget approva for coming $y e a r-c$, Changes in

budget in current year-A, Changes in budget in current year-B,

changes in budget in current year-c, Last 6 months purchased items

-A, Last 6 months purchased items-B, Last 6 months purchased items-

$c$,Project expenses-A, Project expenses-B, Project expenses-c, webmai

probłem-A, webmaj prob7em-B, webmaj 7 problem-C, user Id prob7em-A,

User Id problem-B, User Id problem-c, salaries payrof1 problem-A,

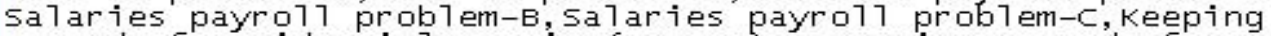

record of residentiaך permit-(AqaamaS-A, keeping record of residentia pi

$r$ internationa 7 students-C, Information sharing prob 7 ems-A,

Information sharing problems-B, Information sharing problems-c,

online transferring of salaries to bank accounts-A, on ine

transferring of saTaries to bank accounts-B, on ine transferring

of sajaries to bank accounts-c, Percentage increment control in

salaries-A, Percentage increment control in salaries-B, Percentage

increment control in sa7aries-C, New employee entry problem-A, New

employee entry problem-B, New employee entry prob7em-C

$N, N, Y, N, Y, N, N, N, Y, N, N, N, Y, N, N, N, Y, N, N, N, N, Y, N, N, Y, N, N, N, Y, N, N, N, Y, N, N, N$ $Y, N, N, N, N, N, N, Y, Y, N, N, N, N, Y, N, N, Y, N, N, N, Y, N, N, N, N, Y, N, N, N, Y, N, N, Y, N, N, N$ $N, N, N, Y, Y, N, N, N, N, Y, N, N, Y, N, N, N, N, Y, N, N, N, N, N, Y, Y, N, N, N, Y, N, N, N, Y, N, N, N$ $Y, N, N, N, Y, N, N, N, Y, N, N, N, Y, N, N, N, Y, N, N, N, Y, N, N, Y, N, Y, N, Y, N, N, Y, N, Y, N, N, N$ $N, Y, N, N, N, N, Y, N, Y, N, N, N, N, N, N, Y, N, Y, N, N, Y, N, N, N, Y, N, N, N, Y, N, N, N, Y, N, N, N$

\section{Fig. 4- Input File for Apriori}

\section{Rules Generation - Notepad}

File Edit Format View Help

RuTe-1:Rejected

Rule-2:selected

RuTe-3:Rejected

Rule-4 : selected

Rule-5: selected

Rule-6:selected

Rule-7:Rejected

Rule-8:Rejected

Rule-9:selected

Rule-10: selected

Rule-11:Rejected

RuTe-12:Rejected

Rule-13: selected

Rule-14 : selected

Rule-15:Rejected

Rule-16:selected

Rule-17: selected

Rule-18:Rejected

Changes in contracts for New Employee ---> Employee ID Probl website Problem ---> online Registration Problem, Login prob online Registration Probelm $--\rightarrow$ Mail server Problem website Problem ---> Downloading and uploading Problem

college Portal problem ---> Faculty Record Maintenance probl Salary Payrol1 Problem ---> Percentage Increment Problem Information sharing Problem ---> University Magazine Passport Keeping Record Problem ---> Iqaama Issue Problem New Employees Entries ---> Passport Keeping Record Problem Changes in Contracts for New Employee -- $\rightarrow$ Employee ID Probi Project Expenses Problem ---> Equipment Problem Changes in Budget ---> Project Expenses Problem Changes in Budget------> Project Expences Problem, Equipment Bugs in software ---> salary calculation Mistake, Employee I Login Problem ---> Mail server Problem webmail Problem ---> Login problem, Mail server Problem Database problem ---> Information Sharing Problem, Salary Tr Changes in Contracts for New Employee ---> salary Problem

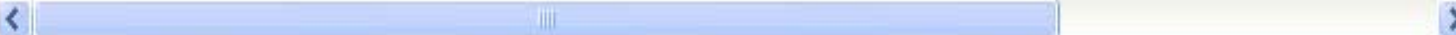

\subsection{Rules Generation}

Fig. 5- Rules Generated File

We applied Apriori algorithm for the generation of frequent itemsets. These Itemset generation is based on the customer query, corresponding binary value, minimum support $(5 \%)$ and minimum confidence $(70 \%)$. Fig.5 is show the number of rules generated by running the Apriori algorithm. This was a sample 
implementation of Apriori algorithm on the presented model. We can find different kind of frequent itemsets generation in the same manner by increasing or decreasing the minimum support and confidence. The selected rules then proposed and suggested to the MADAR.

\section{Future Work}

The model will be enhancing with more information and more data mining technique will be applying and new rules will be generated in future for the enhancement of an organization. New ERP tools will be used to modify the existing work and make ease for the customers to access the organization's facilities with out any hesitation.

\section{Conclusion}

In today's technologies the customers have a lot of difficulties to access the organization's facilities. The customer have problem in contacting the organization. The model presented in this paper will solve these problems all the customers complaints will be recording in the central database and will be process according to the customer need. The customer can easily contact the organization and can purchase the organization products very easily. The CRM-Layer will collect the information about the products and the queries will be forwarded to the ERP-Layer to act upon these queries. The knowledge discovery layer generates new rules and patterns for the betterment of an organization for future correspondence to improve the growth of the customers for an organization.

\section{References}

[1] Ryals, L. and Knox, S. (2001) "Cross-Functional Issues in the Implementation of Relationship Marketing Through Customer Relationship Management", Cranfield University School of Management.

[2] Stanley B. (2000), Customer Relationship Management, John Wiley \& Sons Canada Ltd.

[3] Tinsley, Dillard B. (2002) "Relationship marketing s strategic array", Stephen F. Austin State University, Nacogdoches, Texas.

[4]. Wen-Hsiung Wu, Chin-Fu Ho, Hsin-Pin Fu, Tien-Hsiang Chang, "SMES IMPLEMENTING AN INDUSTRY SPECIFIC ERP MODEL USING A
CASE STUDY APPROACH", Journal of the Chinese Institute of Industrial Engineers, Vol. 23, No. 5, 2006, pp. 423-434.

[5]. Virgil Chichernea, Romanian, "THE USE OF THE ERP-CRM-CIM SYSTEMS WITHIN THE MASTER'S DEGREE PROGRAMMES"

[6].Executive guidance, "http://www.netessence. com.cy/downloads/erp.pdf" , Accessed date: March 21, 2009.

[7].Apriori Algorithm, http://en.wikipedia.Org /wiki/Apriori algorithm \#Algorithm,

Accessed Date: 12th April, 2009.

[8]. Agrawal R, Imielinski T, Swami AN. "Mining Association Rules between Sets of Items in Large Databases." SIGMOD. June 1993, 22(2):207-16.

[9]. Sonja Grabner-Kraeuter, Gernot Moedritscher, Martin Waigunyc, Werner Mussnigb, "Performance Monitoring of CRM Initiatives", in Proceedings: IEEE conference on System Sciences, 2007.

[10]. Mahdi Seify, "New Method for Risk Management in CRM Security Management", in proceedings: IEEE conference on Information Technology, 2006.

[11] S. Kotsiantis, D. Kanellopoulos, Association Rules Mining: A Recent Overview, GESTS International Transactions on Computer Science and Engineering, Vol.32 (1), 2006, pp. 71-82.

[12] Raza Ali, Usman Ghani, Aasim Saeed, "Data Clustering and Its Applications", http:// members.tripod.com/asimsaeed/paper.htm, Accessed Date, 15th April, 2009.

[13] Anil K. Jain, "Data Clustering: 50 Years Beyond K-Means", $19^{\text {th }}$ International Conference Pattern Recognition (ICPR), Tampa, FL, December 8, 2008.

[14]. Abdullah Al- Mudimigh, Farrukh Saleem, Zahid Ullah, "Efficient Implementation Of Data Mining: Improve Customer's Behavior", The 7th ACS/IEEE International Conference on Computer Systems and Applications, Morocco, May 10, 2009, pp-7-10. 